\title{
Commissioning of the ATLAS high-level trigger with single beam and cosmic rays
}

\author{
V. Erkcan Özcan, on behalf of the ATLAS Collaboration*,a \\ ${ }^{a}$ University College London, Dept. of Physics and Astronomy, Gower St., London, WC1E 6BT, U.K.
}

\begin{abstract}
ATLAS is one of the two general-purpose detectors at the Large Hadron Collider (LHC). Using fast reconstruction algorithms, its trigger system needs to efficiently reject a huge rate of background events and still select potentially interesting ones with good efficiency. After a first processing level using custom electronics, the trigger selection is made by software running on two processor farms, designed to have a total of around two thousand multi-core machines. This system is known as the High Level Trigger (HLT). To reduce the network data traffic and the processing time to manageable levels, the HLT uses seeded, step-wise reconstruction, aiming at the earliest possible rejection of background events.

The recent LHC startup and short single-beam run provided a "stress test" of the trigger. Following this period, ATLAS continued to collect cosmic-ray events for detector alignment and calibration purposes. These running periods allowed strict tests of the HLT reconstruction and selection algorithms as well as of its configuration and monitoring systems. This facilitated the commissioning of several tracking, muon-finding, and calorimetry algorithms under different running conditions.

After an overview of the trigger design, this paper focuses on the experience gained in running the trigger in the fast-changing environment of the detector commissioning. It will emphasize the commissioning of the HLT algorithms, monitoring and configuration.
\end{abstract}

Key words:

ATLAS commissioning, High-level trigger

\section{Introduction}

ATLAS, described in Ref. [1], is one of the two generalpurpose detectors at the Large Hadron Collider (LHC). With the LHC operating under design luminosity and $14 \mathrm{TeV}$ center-of-mass energy, ATLAS will see 40 million events per second with about 25 overlapping interactions. Most of these events being inelastic proton-proton collisions, ATLAS has to select about one event to be stored out of $10^{5}$, while keeping the potentially interesting events with good efficiency. This is achieved by a three-level trigger system, the first of which (L1) is based on custom hardware. L1, using coarse data from the calorimeter and muon subdetectors, reduces the rate by $\sim 3$ orders of magnitude with a latency of less than $2.5 \mu \mathrm{s}$.

Running in "regions-of-interest" defined by L1 is the second-level (L2) trigger, which executes fast dedicated reconstruction algorithms on data from all subdetectors at full granularity. L2 is designed to reduce to trigger rate down to $\sim 3 \mathrm{KHz}$. It is the task of the Event Filter (EF), the third-level trigger which runs offline algorithms, to reduce the rate further to the event recording rate of

\footnotetext{
* Corresponding author. Tel.: +41-22-767-8733.

Email address: eo@hep.ucl.ac.uk (V. Erkcan Özcan, on behalf of the ATLAS Collaboration)
}

$\sim 200 \mathrm{~Hz}$. L2 and EF are software-based triggers running on commercial PCs and together comprise the High-Level Trigger (HLT). L2 (EF) has been designed to have a mean processing time below $40 \mathrm{~ms}(4 \mathrm{~s})$.

Currently 842 nodes (each with dual Harpertown quadcore CPUs), out of a planned total of about two thousand, have been installed on 27 racks and are being maintained by a homegrown tool based on Nagios [2]. Each of the racks are configurable to run L2 or EF software, allowing various stress tests of the system.

\section{HLT Software Architecture and Operation}

The main units of the HLT are algorithms that extract features (clusters, tracks, etc.) and apply requirements on them (for example, matching the clusters to tracks). Algorithms are organized in sequences that produce potential candidates for interesting objects such as high-transversemomentum electrons, photons, taus, muons, ( $b$-tagged) jets, missing energy, or signatures of $B$-physics or minimumbias events. A set of sequences, along with the L1 items that initiate them and the signatures obtained by various combinations of their results, comprise the trigger menu. The menu definition also includes the parameters of all the underlying algorithms and the pass-through and prescale fractions. 
The execution of the system is handled by the HLT steering, which provides two essential features: Caching of all results prevents redundant rerunning of any of the steps. Early rejection cancels execution of any unnecessary steps as soon as a sequence fails.

The menu, the identifiers for the particular versions of its constituent algorithms, and the bunch numbers for collisions are recorded in a relational database with many-tomany links that prevents any duplication of objects. Any given configuration is readily represented by only 4 keys, which allows the run control to easily change the passthrough and prescale factors at luminosity block boundaries, and the complete menu at any run change. Experts and shifters can modify the menu (or parts of it, depending on access priviledges) using a Java-based tool. This tool creates new keys as needed and keeps a history of all the configurations, which is accessible to the offline users on a read-only basis.

The trigger is monitored online and offline. Online monitoring includes the monitoring of the rates and data quality, both by the shifters and automatically, and an event display which displays both offline-reconstructed and trigger objects at a rate of $\sim 1 \mathrm{~Hz}$. Offline monitoring allows reviewing of the saved trigger quantities and their comparison to the output of fast offline reconstruction performed at Tier-0 at CERN. This system, comprised of 1600 cores of CERN batch machines, is designed to reconstruct all events from ATLAS within 24 hours.

The trigger software to be deployed on the HLT farms goes through intensive checks for stability. For this purpose, a patch branch is maintained separately from the regular development branch of the code, with its own nightly test cycles that run on recently recorded data. 64 cores are reserved exclusively for these trigger tests at the CERN Analysis Facility, a center dedicated to run on about $10 \%$ of all the recorded events for commissioning and calibration of the detector. This facility also handles events that go into the debug stream, ie. those events with HLT errors or timeouts.

\section{Commissioning with Data}

For the first beams in the LHC, the priorities for ATLAS were stability and reliability. For this purpose, silicon detectors were turned off or ran at low-bias voltage, and a simple trigger configuration based on only the L1 decisions was selected. The HLT was used only for the tagging of the events and for routing them into data streams. Algorithms, except those crucial for the routing task, were not exercised. The data collected during the short run with the beams were mostly useful for the timing of the L1 triggers, in particular the timing of the signals from the beam pick-up system (BPTX) and the minimum-bias trigger scintillators (MBTS). HLT algorithms were later tested offline on those events with muon and calorimeter regions-of-interest in time with the BPTX or the MBTS, but due to limited statistics (less than a thousand events), such tests were limited mostly related to basic functionality.

A more complete test of the HLT was possible during the subsequent cosmic ray runs. In 2008, starting from September 13th, ATLAS collected 216 million events until the end of October, and more than 100 million events after that. While many cosmic runs had been taken in the recent years as part of the detector commissioning, these new runs presented the first opportunity to fully exercise the detector, including the recently installed pixel detector.

Cosmic rays present particular challenges to the HLT, which has been designed to perform best for collision events. Since no LHC clock is present, the timing of the event is provided by the muon trigger chambers and the corresponding jitter can adversely affect the readout of some of the subdetectors, particularly those which rely on the drift of charge carriers, such as the Transistion Radiation Tracker (TRT) or the Monitored Drift Tube (MDT, precision muon) chambers. Furthermore, L2 algorithms for fast tracking in the inner detector and the muon spectrometer normally require that the tracks point towards the beam axis and have small impact parameters. Neither of these requirements are satisfied for most of the cosmic tracks. Finally, cosmic muons rarely provide the kind of signatures that initiate the chains associated with non-muon physics objects in the trigger menu.

Despite these, an effort was made to exercise all the chains. By relaxing various thresholds and requirements, both L2 and EF algorithms from all groups of trigger objects were successfully run and tested. An example output from the electron-photon feature extraction is shown in Fig. 1. Most extensive studies were possible for the muon reconstruction, for which the high statistics obtained by relaxing the pointing requirement allowed studies of efficiency and rejection. For instance, the endcap MDT cluster finding efficiency in the L2 was found to be $93 \%$, with almost all of the inefficiency identified to be due to detector or timing issues.

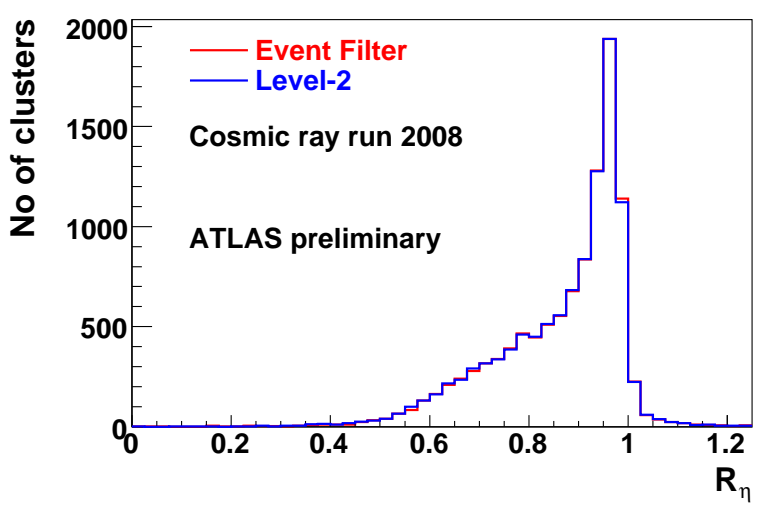

Figure 1: The distribution of $R_{\eta}$ (sometimes also denoted by $R_{\text {core }}$ ), the shower shape in $\eta$ direction in the second EM sampling. A measure of the narrowness of EM clusters, it is described in detail in Ref [3]. The histograms from L2 and EF lie on top of each other in excellent agreement. 
Beyond these exercises towards the commissioning of the trigger system itself, HLT played an important role in the commissioning of the ATLAS inner detector (pixel and microstrip silicon detectors, and the TRT). Only a small percentage of the cosmic muons that trigger the L1 muon chambers transverse the inner detector (ID) and the high L1-muon rate $(\sim 500 \mathrm{~Hz})$ denies the recording of all muon events. A fast and proper alignment of the ID requires as many tracks as possible, with no biases in their selection. Since ID tracking is not possible at L1, an essential task for the L2 tracking during the cosmic runs is to provide the needed tracks.

This task requires significant modifications to the three L2 tracking algorithms. To impart a sense of the level of modifications needed, it can be pointed out that the design of the two $\mathrm{Si}$ algorithms (those that take hits from the pixel and microstrip sensors as input) is such that the efficiency drops dramatically for tracks with transverse impact parameter $\left(d_{0}\right)$ beyond a few millimeters, while for the cosmic tracks good efficiency is desired up to hundreds of $\mathrm{mm}$. Another issue arises due to the presence of noisy modules that produce tens of fake hits: with no pointing regions-of-interest, the algorithms have to run with data from the whole detector and in an otherwise very clean environment with only a few hits from the cosmic tracks, such noise hits can abnormally damage the performance unless they are taken into account.

Therefore, new cosmic chains were defined to be run in parallel to the regular trigger menu. These chains were initiated by any L1 signature (muon, calorimeter, MBTS) and ran the modified L2 tracking algorithms. For the TRT-based tracking, pattern recognition was completely independent from the one designed for collision data, but the same data preparation and software machinery were used. Modifications to one of the $\mathrm{Si}$ algorithms allowed track seeds to be created anywhere in the Si detectors. The other Si algorithm was run with settings relatively close to the collision values, but a separate pattern recognition developed for cosmic rays shifted the hits in the entire Si detector to account for the high impact parameter. Overall, effort was made to exercise as much of the regular L2 software as possible.

Fig. 2 shows the number of collected tracks from cosmic rays that trespassed the pixel detector. It should be highlighted that a good-level of robustness had been achieved by the end of October and when the ID was reincorporated into the global run following a month-long break, HLT tracking was running very smoothly despite some changes to the detector configuration. Measurements of the collection efficiency for events containing "golden silicon" tracks (offline tracks with at least 3 hits in the upper and at least 3 hits in the lower barrel of the Si detectors), indicate that more than $99 \%$ of such events were retained by at least one of the three algorithms.

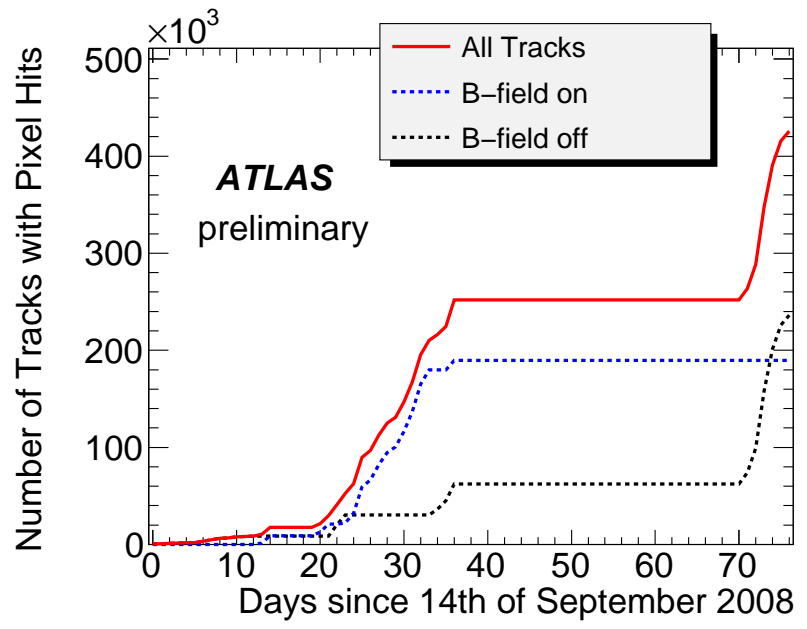

Figure 2: Number of collected cosmic-ray tracks with pixel-detector hits as a function of time. Most of the data collected until the end of October was with the magnetic field on, whereas for the data collected at the end of November, the field was off.

\section{Conclusion}

All of the HLT infrastructure (L2 and EF algorithms, steering, streaming and monitoring) was exercised during the last months of 2008 and was tested to work under actual datataking conditions. It played a crucial role in collecting the inner detector tracks that were necessary for the detector alignment. Studies of the muon and calorimeter signatures provided useful feedback to those detector systems and played a role in the online monitoring of the data quality. Finally HLT infrastructure was used for tagging data and diverting it into the relevant streams.

The overall process was also a test of the HLT operation cycle. Commissioning was performed while HLT was serving the subsystems; the issues of running on actual data were resolved as the datataking was continuing. A good balance was achieved between stability and responsiveness to the requests of the subsystems and to the detector conditions.

Some work that is still going on as we approach the restarting of the LHC include: advanced monitoring tools that make more use of the comparisons of trigger and offline reconstruction, speeding up the boot-up and configure times, and additional studies of the cosmic-ray performance to gain insights to the expected performance with LHC collisions. In the coming months before the scheduled restart of the LHC, further cosmic-ray data will be collected and additional stress tests of the system will be performed using Monte Carlo data injected to the HLT.

ATLAS is looking forward to the LHC collision data.

\section{References}

[1] The ATLAS Collaboration, G. Aad et al., JINST 3 (2008) S08003.

[2] Nagios Enterprises, LLC, http://www.nagios.org/

[3] The ATLAS Collaboration, CERN-OPEN-2008-020, 2008. 